\title{
Clinical Benefits of Microscopically Complete Resection in Controlling Local Recurrence of Single Brain Metastasis
}

\author{
Ji Hwan Jang, Young Min Lee and Young Zoon Kim \\ Department of Neurosurgery, Sungkyunkwan University School of Medicine, Samsung Changwon Hospital, Changwon, Korea
}

\section{Abstract}

Objective:The influence on therapeutic outcomes of wide surgical resection of brain metastasis (BM) including tumor cells that microscopically infiltrate adjacent brain parenchyma, with clean surgical margins confirmed by intraoperative margin biopsy is not known. This study was carried out to clarify the therapeutic impact of these resections.

Methods: From June 2002 to April 2014, 90 patients (66 men and 24 women; mean age: 56.7 years) underwent surgical resection for BM. In 47 patients (group A), we undertook microscopically complete resection, including microscopic infiltrative tumor cells adjacent to the brain parenchyma, with pathologically clean surgical margins confirmed during surgery for BMs in a noneloquent area. In 43 patients (group B), we undertook gross complete resection without removal of neighboring brain parenchyma due to eloquent location. The two groups were compared for survival, and local and distant recurrence.

Results:Mean follow-up duration was 18.6 months; 71 patients (78.9\%) died during follow-up. Median survival was 13.1 months in group $A$ and 12.1 months in group $B(p=0.309)$. One-year and two-year local recurrence were $21.2 \%$ and $25.8 \%$, respectively, in group $A$, and $65.2 \%$ for both in group $B(p=0.000)$. One-year and two-year distant recurrence rates were $46.3 \%$ and $57.2 \%$ in group A, respectively, and $33.2 \%$ for both in group $\mathrm{B}(\mathrm{p}=0.140)$.

Conclusion: These results suggested that wide surgical resection of BM including tumor cells infiltrating adjacent brain parenchyma with confirmed clean surgical marginsresulted in better local control.

\author{
Publication History: \\ Received: June 28, 2016 \\ Accepted: August 04, 2016 \\ Published: August 06, 2016
}

\section{Keywords:}

Microscopical complete resection, Brain metastasis, Local control, Survival, Surgical resection, Margin biopsy, Neurosurgery

\section{Introduction}

An estimated 98,000 to 170,000 new cases of brain metastasis (BM) are diagnosed in the United States each year although the true incidence is not known. Variations in estimates of incidence and prevalence arise from differences in the method of assessment [1]. In the largest autopsy series to date, Posner and Chernik examined the records of 2375 cancer patients and found that central nervous system metastases occur in approximately $24 \%$ of patients with systemic cancer [2].

Some patients with BM can be treated with craniotomy for palliative purposes, usually combined with brain irradiation. When potential candidates are considered for resection, determining who will benefit from surgical resection and postresection treatment is difficult because of the limited survival time associated with BM. However, surgery for BM provides certain advantages over other therapeutic modalities [3]. First, the excision of a metastasis can immediately eliminate the effects of increased intracranial pressure and direct symptomatic effects on surrounding brain tissues. Second, surgery provides tissue for confirming a diagnosis of metastasis. Finally, surgery may establish a local cure or complete remission if all tumor cells can be removed.

Recent advances in microsurgical techniquessuch as navigation systems, intraoperative ultrasonography, and cortical mapping have promoted safe surgical resection; this is particularly the case for single lesions, which account for $40-60 \%$ of all BMs $[4,5]$. Modern imaging techniques can now locate and define smaller metastases that can be operated on earlier in their development.

However, despite the advantages of surgery for BM, this approach is not fully accepted because of the high tumor recurrence rate after BM resection [6]. Even if complete resection is confirmed by both intraoperative evaluation and immediate postoperative magnetic resonance (MR) imaging, increased patient survival inevitably increases the number of recurrences due to residual foci of microscopic and infiltrative tumors left after surgery, particularly in eloquent areas of the brain.Attempts to achieve clean surgical margins increase the risk of neurological dysfunction. Microscopically, small nests and trabeculae of tumor cells can be seen at tumor borders, infiltrating adjacent nervous tissue. However, these features do not typically extend more than $5 \mathrm{~mm}$ beyond the circumference of a solid tumor [7].

To reduce local recurrence for tumors located in noneloquent areas, we undertook wide surgical resection of BMs including microscopic, infiltrative tumor cells adjacent to the brain parenchyma, confirming clean surgical margins by intraoperative margin biopsy. For tumors located in an eloquent area, we undertook gross complete resection without additional removal of surrounding brain parenchyma. We evaluated the validity of wide surgical resection of BM including tumor cells microscopically infiltrating adjacent brain parenchyma by comparing the two patient groups for local and distant recurrence rates and overall survival.

"Corresponding Author: Prof. Young Zoon Kim, Division of Neurooncology and Department of Neurosurgery Sungkyunkwan University School of Medicine, Samsung Changwon Hospital 158 Paryong-ro, Masanhoewon-gu, Changwon, 51353, Korea, Tel. +82-55-2335873; E-mail: yzkim@skku.edu

Citation: Jang JH, Lee YM, Kim YZ (2016) Clinical Benefits of Microscopically Complete Resection in Controlling Local Recurrence of Single Brain Metastasis. Int J Surg Surgical Porced 1: 108. doi: http://dx.doi.org/10.15344/ijssp/2016/108

Copyright: @ 2016 Jang et al. This is an open-access article distributed under the terms of the Creative Commons Attribution License, which permits unrestricted use, distribution, and reproduction in any medium, provided the original author and source are credited. 
Citation: Jang JH, Lee YM, Kim YZ (2016) Clinical Benefits of Microscopically Complete Resection in Controlling Local Recurrence of Single Brain Metastasis. Int J Surg Surgical Porced 1: 108. doi: http://dx.doi.org/10.15344/ijssp/2016/108

Page 2 of 9

\section{Materials and Methods}

\section{Study design}

Between June 2002 and April 2014, 90 patients underwent craniotomy at our institute to remove a BM. Of these, 47 (group A) underwent wide surgical resection of $\mathrm{BM}$ including microscopic, infiltrative tumor cells adjacent to brain parenchyma with a 5-mm tumor-free margin outside tumor capsules. Clean surgical marginswere confirmed by marginal biopsy during surgery. After identifying the gliotic pseudocapsule surrounding the tumor through a transcortical or transsulcal approach, we attempted en bloc resection without rupturing the tumor compartment, if possible. After gross total removal of the tumor, we resected additional surrounding brain tissue to a depth of at least 5-mm until a pathological free margin was confirmed. We defined the above pattern of surgical resection of BM as microscopically complete resection (MCR). Another group of 43 patients (group B) underwent gross complete resection (GCR) of BM without additional resection of adjacent brain parenchyma due to the eloquent location.

\section{Clinical characteristics of patients}

Retrospective analysis of survival, local recurrence and distant recurrence in relation to clinical variables was performed. Variables were patient's age and gender, type of primary cancer, extracranial metastasis, status of primary cancer, Karnofsky Performance Scale (KPS) scores and recursive partitioning analysis (RPA) class.

According to the classification described by Gasper et al. [8], patients in RPA Class 1 were characterized by age $<65$ years, KPS score $\geq 70$ and absence of extracranial metastases, and good control of systemic disease. RPA Class 2 patients had KPS score $\geq 70$, but may also be aged $\geq 65$ years, or have uncontrolled systemic disease or other systemic metastases. Patients in RPA Class 3 had KPS $<70$.

\section{Features of brain metastases}

Variables were timing of metastasis, tumor size, tumor location, functional grade of lesion and method of tumor resection. Brain metastasis diagnosed $<60$ days from primary lesion diagnosis was considered synchronous; diagnosis 60 days from primary cancer diagnosis was defined as metachronous. Tumor size was determined as maximal orthogonal diameter and divided into $<3 \mathrm{~cm}$ and $\geq 3 \mathrm{~cm}$. Tumor location was categorized as supratentorial or infratentorial. Using preoperative MR imaging, tumorswere functionally graded by location relative to eloquent brain according to a scheme developed at M.D. Anderson Cancer Center [9]. Functional grade I tumors were in noneloquent brain (e.g., frontal or temporal polar lesion), grade II tumors in near-eloquent brain (e.g., motor or sensory cortex, calcarine fissure, speech center, dentate nucleus, brain stem or corpus callosum) and grade III tumors were in eloquent brain (e.g., motor and sensory cortex, visual center, speech center, internal capsule, basal ganglia, hypothalamus, thalamus, brain stem and dentate nucleus).

\section{Surgical indications}

Clinical indications were symptoms and signs of intracranial hypertension unresponsive to adequate medical therapy (e.g., corticosteroid and mannitol), intractable seizures, reduced level of consciousness, progressive motor weakness, gait ataxia and aphasia. Neuroimaging indications were lesion enlargement, hemorrhage, and mass effect from edema unresponsive to maximal medical therapy.

\section{Outcomes and data analysis}

Survival times were defined from date of surgical resection until death or last day of follow-up. Postsurgical imaging studies were reviewed for evidence of central nervous system (CNS) recurrence. Local recurrences were defined as within $2 \mathrm{~cm}$ of initial tumor resection bed. Distant recurrences in the CNS were defined as any of the following: $>2 \mathrm{~cm}$ from initial surgical site, on the contralateral side, or in the posterior fossa, spinal cord, or cerebrospinal fluid. For patients who died, cause of death and date of death were recorded. Medical records of all patients were analyzed for clinical history, operative and pathology reports and radiological studies.Dates of death were confirmed for all patients who died.

\section{Statistical analysis}

Statistical analyses used SSPS version 12.0 (SPSS Institute, Inc., Chicago, IL). Progression-free-survival (PFS) and overall survival were estimated using Kaplan-Meier methods (Figure 1). Log-rank test and Cox regression analysis were used to examine progressionfree and overall survival. Variables significantly associated with survival by univariate analyses were subject to multivariate analyses. T-tests and chi-square tests were used to compare groups by baseline characteristics. Results were considered significant when p-values were less than 0.05 .

\section{Results}

\section{Patient population}

Table 1 summarizes patient characteristics. Group A had 35 men and 12 women of mean age 57.9 years (range: 14-84) when brain metastasis was diagnosed; group B had 31 men and 12 women of mean age 55.3 years (range: 12-77) at diagnosis. The histological distribution of primary tumor types was: 66 non-small cell lung cancers, 3 small cell lung cancers, 7 colorectal cancers, 6 renal cell cancers, and 11 other systemic cancers.

A total of 49 patients (54.4\%) had extracranial metastases, 28 $(59.6 \%)$ in group A and $21(48.8 \%)$ in group B, with 56 cancers (62.2\%) under control and $28(37.8 \%)$ progressing. A total of 62 patients $(68.9 \%)$ had KPS scores $\geq 70,39(83.0 \%)$ in group A and 23 $(53.5 \%)$ in group B. In group A, 10 patients $(21.3 \%)$ were in RPA class I, $32(68.1 \%)$ in RPA class II and $5(10.6 \%)$ in RPA class III. In group B, 6 patients (14.0\%) were in RPA class I, 17 (39.5\%) in RPA class II, and 20 (46.5\%) in RPA class III.

No significant differenceswere observed between the two groups in demographic parameters or patient features. However, group A had higher KPS scores $(p=0.003)$ and lower RPA classes $(p=0.001)$ than group B.

\section{Features of brain metastasis}

Table 2 shows features of brain lesions. A total of 56 patients (25 in group A and 31 in group B) had metachronous development; the others (22 in group A and 12 in group B) had synchronous development. A total of 23 tumors had functional grade 1 (22 in group A and 1 in group B), 45 had functional grade 2 (18 in group A and 7 in group B) and 22 had functional grade 3 (7 in group A and 15 in group $\mathrm{B})(\mathrm{p}=0.000)$. A total of 29 patients, $4(8.5 \%)$ in group A and $25(58.1 \%)$ in group $B$, received radiotherapy after BM surgery 
Citation: Jang JH, Lee YM, Kim YZ (2016) Clinical Benefits of Microscopically Complete Resection in Controlling Local Recurrence of Single Brain Metastasis. Int J Surg Surgical Porced 1: 108. doi: http://dx.doi.org/10.15344/ijssp/2016/108

Page 3 of 9

\begin{tabular}{|c|c|c|c|c|c|}
\hline & & Total & $\begin{array}{l}\text { group A } \\
(\mathrm{N}=90)\end{array}$ & $\begin{array}{l}\text { group B } \\
(\mathrm{N}=47)\end{array}$ & $\begin{array}{l}\mathrm{p} \text { value } \\
(\mathrm{N}=43)\end{array}$ \\
\hline \multirow[t]{2}{*}{ Gender } & male & $66(73.3 \%)$ & $35(74.5 \%)$ & $31(72.1 \%)$ & \multirow[t]{2}{*}{0.799} \\
\hline & female & $24(26.7 \%)$ & $12(25.5 \%)$ & $12(27.1 \%)$ & \\
\hline \multirow[t]{4}{*}{ Age } & mean & 56.66 & 57.87 & 55.33 & \multirow[t]{2}{*}{0.335} \\
\hline & range & $(12-84)$ & $(14-84)$ & $(12-77)$ & \\
\hline & $\geq 65$ & $23(25.6 \%)$ & $16(34.0 \%)$ & $7(16.3 \%)$ & \multirow[t]{2}{*}{0.054} \\
\hline & $<65$ & $67(74.4 \%)$ & $31(66.0 \%)$ & $36(83.7 \%)$ & \\
\hline \multirow[t]{9}{*}{ Primary cancer } & NSCLC $^{*}$ & $63(70.0 \%)$ & $34(72.3 \%)$ & $29(67.4 \%)$ & 0.612 \\
\hline & SCLC $\dagger$ & $3(3.3 \%)$ & $3(6.4 \%)$ & $0(0.0 \%)$ & \\
\hline & colorectal & $7(7.8 \%)$ & $3(6.4 \%)$ & $4(9.3 \%)$ & \\
\hline & kidney & $6(6.7 \%)$ & $3(6.4 \%)$ & $3(7.0 \%)$ & \\
\hline & gynecology & $3(3.3 \%)$ & $1(2.1 \%)$ & $2(4.7 \%)$ & \\
\hline & liver & $3(3.3 \%)$ & $0(0.0 \%)$ & $1(2.3 \%)$ & \\
\hline & breast & $2(2.2 \%)$ & $2(4.3 \%)$ & $2(4.7 \%)$ & \\
\hline & stomach & $2(2.2 \%)$ & $1(2.1 \%)$ & $1(2.3 \%)$ & \\
\hline & others & $1(1.1 \%)$ & $0(0.0 \%)$ & $1(2.3 \%)$ & \\
\hline \multirow[t]{2}{*}{ Extracranial metastasis } & presence & $49(54.4 \%)$ & $28(59.6 \%)$ & $21(48.8 \%)$ & \multirow[t]{2}{*}{0.307} \\
\hline & absence & $41(45.6 \%)$ & $19(40.4 \%)$ & $22(51.2 \%)$ & \\
\hline \multirow[t]{2}{*}{ Status of cancer } & under-control & $56(62.2 \%)$ & $29(61.7 \%)$ & $27(62.8 \%)$ & \multirow[t]{2}{*}{0.915} \\
\hline & out-of-control & $34(37.8 \%)$ & $18(38.3 \%)$ & $16(37.2 \%)$ & \\
\hline \multirow[t]{2}{*}{$\mathrm{KPS}^{\ddagger}$ score } & $\geq 70$ & $62(68.9 \%)$ & $39(83.0 \%)$ & $23(53.5 \%)$ & \multirow[t]{2}{*}{0.003} \\
\hline & $<70$ & $28(31.1 \%)$ & $8(17.0 \%)$ & $20(46.5 \%)$ & \\
\hline \multirow[t]{3}{*}{$\mathrm{RPA}^{\S}$ class } & 1 & $16(17.8 \%)$ & $10(21.3 \%)$ & $6(14.0 \%)$ & \multirow[t]{2}{*}{0.001} \\
\hline & 2 & $49(54.4 \%)$ & $32(68.1 \%)$ & $17(39.5 \%)$ & \\
\hline & 3 & $25(27.8 \%)$ & $5(10.6 \%)$ & $20(46.5 \%)$ & \\
\hline
\end{tabular}

Table 1: Clinical characteristics of the patients according to group A and B.

Note: NSCLC ${ }^{*}$ : non-small cell lung cancer, SCLC ${ }^{\dagger}$ : small cell lung cancer, KPS ${ }^{\ddagger}$ : Karnofsky Performance Scale, RPA Analysis

\begin{tabular}{|c|c|c|c|c|c|}
\hline & & Total $(\mathrm{N}=90)$ & group $\mathrm{A}(\mathrm{N}=47)$ & group $\mathrm{B}(\mathrm{N}=43)$ & $\mathrm{p}$ value \\
\hline \multirow{2}{*}{ Timing of metastasis } & synchronous & $34(37.8 \%)$ & $22(46.8 \%)$ & $12(27.9 \%)$ & \multirow[t]{2}{*}{0.065} \\
\hline & metachronous & $56(62.2 \%)$ & $25(53.2 \%)$ & $31(72.1 \%)$ & \\
\hline \multirow[t]{2}{*}{ Tumor size } & $<3 \mathrm{~cm}$ & $31(34.4 \%)$ & $12(25.5 \%)$ & $19(44.2 \%)$ & \multirow[t]{2}{*}{0.063} \\
\hline & $\geq 3 \mathrm{~cm}$ & $59(65.6 \%)$ & $35(74.5 \%)$ & $24(55.8 \%)$ & \\
\hline \multirow[t]{2}{*}{ Tumor location } & supratentorial & $75(83.3 \%)$ & $37(78.7 \%)$ & $38(88.4 \%)$ & \multirow[t]{2}{*}{0.220} \\
\hline & infratentorial & $15(16.7 \%)$ & $10(21.3 \%)$ & $5(11.6 \%)$ & \\
\hline \multirow[t]{3}{*}{ Functional grade } & 1 & $23(25.6 \%)$ & $22(46.8 \%)$ & $1(2.3 \%)$ & \multirow[t]{2}{*}{0.000} \\
\hline & 2 & $45(50.0 \%)$ & $18(38.3 \%)$ & $27(62.8 \%)$ & \\
\hline & 3 & $22(24.1 \%)$ & $7(14.9 \%)$ & $15(34.9 \%)$ & \\
\hline \multirow[t]{2}{*}{ Postoperative radiotherapy } & performed & $29(32.2 \%)$ & $4(8.5 \%)$ & $25(58.1 \%)$ & \multirow[t]{2}{*}{0.000} \\
\hline & not-performed & $61(67.8 \%)$ & $43(91.5 \%)$ & $18(41.9 \%)$ & \\
\hline
\end{tabular}

Table 2: Features of the brain metastasis according to group A and B.

$(\mathrm{p}=0.000)$. Comparing features of brain metastasis showed significant differences between groups fortumor functional grade $(\mathrm{p}=0.000)$ and postoperative radiotherapy $(\mathrm{p}=0.000)$.

\section{Postoperative Follow-up}

No patients were lost to follow-up. The absence of residual enhancing lesions was confirmed by immediate postoperative MRI within 48 hours of surgery. All patients underwent a regular MRI study at one month after surgery and then every 3 months or when clinically indicated. Overall median survival time (MST) for the all patients was 12.4 months (range: $3.2-56.8$ ). In group A, MST was 13.1 months (range: 3.3-56.8) and in group B, 12.1 months (range: 3.2-47.8). 
Citation: Jang JH, Lee YM, Kim YZ (2016) Clinical Benefits of Microscopically Complete Resection in Controlling Local Recurrence of Single Brain Metastasis. Int J Surg Surgical Porced 1: 108. doi: http://dx.doi.org/10.15344/ijssp/2016/108

Page 4 of 9

During the follow-up period, 71 (78.9\%) patients died, 35 (74.5\%) in group A and 36 (83.7\%) in group B. Most died of the systemic cause of progression of primary cancer or complication of primary disease. Four patients died from CNS problems, one had a massive hypertensive intracerebral hemorrhage in a location remote from the brain metastasis at 124 days after surgery, and the other three patients died of meningeal carcinomatosis. Formally, three patients (3.3\%) died from a cause of CNS origin.

\section{Survival analysis}

Table 3 summarizes survival analysis by group. MST was calculated from date of surgery for BM. MST was 13.1 months (range: 3.3-56.8) for group A and 12.1 months (range: 3.2-47.8) for group B. One-year survival was $55.2 \%$ in group A and $50.6 \%$ in group B. Two-year survival was $25.6 \%$ in group A and $10.7 \%$ in group B. No significant difference was observed in median survival or 1-year or 2-year survival rate $(\mathrm{p}=0.309)$. Cox regression analysis showed no difference in hazard ratio between the two groups. Overall survival and differencesin survival between the two groups according to follow-up, determined by Kaplan-Meier survival curves, are in Figure 1.

\begin{tabular}{|l|l|l|l|}
\hline & $\begin{array}{l}\text { Total } \\
(\mathrm{N}=90)\end{array}$ & $\begin{array}{l}\text { group A } \\
(\mathrm{N}=47)\end{array}$ & $\begin{array}{l}\text { group B (N } \\
=43)\end{array}$ \\
\hline Mean survival (month) & 18.6 & 21.1 & 14.6 \\
\hline Median survival (month) & 12.4 & 13.1 & 12.1 \\
\hline Ranges (month) & $3.2-56.8$ & $3.3-56.8$ & $3.2-47.8$ \\
\hline 1-year survival rate & $52.9 \%$ & $55.2 \%$ & $50.6 \%$ \\
\hline 2-year survival rate & $19.3 \%$ & $25.6 \%$ & $10.7 \%$ \\
\hline
\end{tabular}

Table 3: Survival analysis and 1-year and 2-year survival rates according to group $\mathrm{A}$ and $\mathrm{B}$.

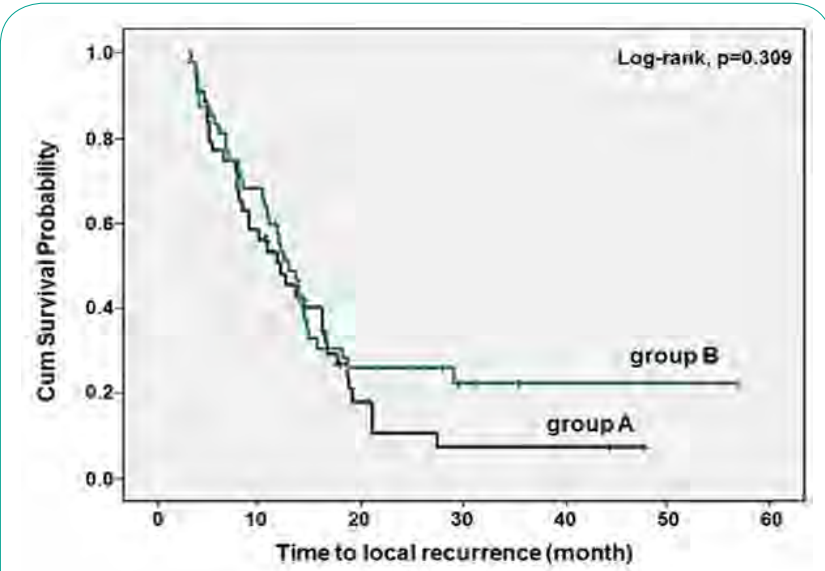

Figure 1: Kaplan-Meier survival curve for groups A and B.

In addition to the method used in surgical resection for BM, we also analyzed associations between survival and gender, age, pathology, tumor stage, extracranial metastasis, primary tumor status, KPS score, timing of metastasis, tumor size and location, functional grade and postoperative radiotherapy.

Clinical variables of female gender, age $<65$ years, and KPS score $\geq 70$ were associated with better survival, as estimated by univariate and multivariate survival analysis (Table 4). No brain lesion factors were associated with survival (Table 5).

\section{Local recurrence}

Patterns of local recurrence according to group are in Table 6. A total of 32 patients $(35.6 \%)$ had local recurrence, 9 (19.1\%) in group

\begin{tabular}{|c|c|c|c|c|c|}
\hline Factor & $\begin{array}{l}\text { median } \\
\text { survival } \\
\text { (month) }\end{array}$ & $\begin{array}{c}\text { univariate } \\
\text { analysis } \\
\text { (p value) }\end{array}$ & $\begin{array}{c}\text { Hazard } \\
\text { ratio } \\
\left(95 \% \mathrm{CI}^{*}\right)\end{array}$ & $\begin{array}{c}\text { multivariate } \\
\text { analysis } \\
\text { (p value) }\end{array}$ & $\begin{array}{l}\text { Hazard } \\
\text { ratio } \\
(95 \% \mathrm{CI})\end{array}$ \\
\hline male & 9.1 & \multirow[t]{2}{*}{0.024} & \multirow{2}{*}{$\begin{array}{c}1.77 \\
(1.07,2.91)\end{array}$} & \multirow[t]{2}{*}{0.013} & \multirow{2}{*}{$\begin{array}{c}1.94 \\
(1.15,3.27)\end{array}$} \\
\hline female & 13.6 & & & & \\
\hline$\geq 65$ years & 10.0 & \multirow[t]{2}{*}{0.033} & \multirow{2}{*}{$\begin{array}{c}1.75 \\
(1.04,2.96)\end{array}$} & \multirow[t]{2}{*}{0.024} & \multirow{2}{*}{$\begin{array}{c}1.89 \\
(1.09,3.28)\end{array}$} \\
\hline$<65$ years & 13.4 & & & & \\
\hline NSCLC $\dagger$ & 11.8 & \multirow[t]{2}{*}{0.083} & \multirow{2}{*}{$\begin{array}{c}1.54 \\
(0.94,2.50)\end{array}$} & \multirow[t]{2}{*}{ NA } & \\
\hline non-NSCLC & 13.6 & & & & \\
\hline presence & 11.7 & \multirow[t]{2}{*}{0.066} & \multirow{2}{*}{$\begin{array}{c}1.56 \\
(0.97,2.50)\end{array}$} & \multirow[t]{2}{*}{ NA } & \\
\hline absence & 13.5 & & & & \\
\hline under-control & 12.3 & \multirow[t]{2}{*}{0.181} & \multirow{2}{*}{$\begin{array}{c}1.39 \\
(0.86,2.26)\end{array}$} & \multirow[t]{2}{*}{ NA } & \\
\hline out-of-control & 13.5 & & & & \\
\hline$\geq 70$ & 13.9 & \multirow[t]{2}{*}{0.003} & \multirow{2}{*}{$\begin{array}{c}2.04 \\
(1.26,3.32)\end{array}$} & \multirow[t]{2}{*}{0.000} & \multirow{2}{*}{$\begin{array}{c}12.76 \\
(3.36,46.43)\end{array}$} \\
\hline$<70$ & 5.6 & & & & \\
\hline performed & 12.1 & \multirow[t]{2}{*}{0.250} & \multirow{2}{*}{$\begin{array}{c}1.33 \\
(0.82,2.16)\end{array}$} & \multirow[t]{2}{*}{0.994} & \multirow{2}{*}{$\begin{array}{c}1.00 \\
(0.54,1.87)\end{array}$} \\
\hline not performed & 12.4 & & & & \\
\hline
\end{tabular}

Table 4: Clinical characteristics of the patients affecting survival in univariate and multivariate analysis (Cox regression model).

$\mathrm{CI}^{\star}$ : confidence interval, NSCLC ${ }^{\dagger}$ : non-small cell lung cancer, NA ${ }^{\ddagger}$ : not assessed, KPS ${ }^{\S}$ Karnofsky Performance Scale. 
Citation: Jang JH, Lee YM, Kim YZ (2016) Clinical Benefits of Microscopically Complete Resection in Controlling Local Recurrence of Single Brain Metastasis. Int J Surg Surgical Porced 1: 108. doi: http://dx.doi.org/10.15344/ijssp/2016/108

Page 4 of 9

\begin{tabular}{|c|c|c|c|c|c|c|}
\hline \multicolumn{2}{|l|}{ Factor } & $\begin{array}{l}\text { median survival } \\
\text { (month) }\end{array}$ & $\begin{array}{l}\text { univariate } \\
\text { analysis } \\
\text { (month) }\end{array}$ & $\begin{array}{l}\text { Hazard } \\
\text { ratio } \\
\left(95 \% \mathrm{CI}^{*}\right)\end{array}$ & $\begin{array}{l}\text { multivariate } \\
\text { analysis } \\
\text { (month) }\end{array}$ & $\begin{array}{l}\text { Hazard } \\
\text { ratio } \\
(95 \% \mathrm{CI})\end{array}$ \\
\hline \multirow[t]{2}{*}{ Timing of metastasis } & synchronous & 13.5 & \multirow[t]{2}{*}{0.398} & \multirow{2}{*}{$\begin{array}{l}1.24 \\
(0.76,2.02)\end{array}$} & \multirow[t]{2}{*}{$\mathrm{NA} \dagger$} & \\
\hline & metachronous & 11.7 & & & & \\
\hline \multirow[t]{2}{*}{ Tumor size } & $<3 \mathrm{~cm}$ & 13.9 & \multirow[t]{2}{*}{0.968} & \multirow[t]{2}{*}{$1.01(0.62,1.64)$} & \multirow[t]{2}{*}{ NA } & \\
\hline & $\geq 3 \mathrm{~cm}$ & 12.1 & & & & \\
\hline \multirow[t]{2}{*}{ Tumor locations } & supratentorial & 13.1 & \multirow[t]{2}{*}{0.465} & \multirow{2}{*}{$\begin{array}{l}1.26 \\
(0.68,2.35)\end{array}$} & \multirow[t]{2}{*}{ NA } & \\
\hline & infratentorial & 10.3 & & & & \\
\hline \multirow[t]{2}{*}{ Method of surgery } & Group A (MCR $\ddagger)$ & 13.1 & \multirow[t]{2}{*}{0.309} & \multirow{2}{*}{$\begin{array}{l}1.27 \\
(0.80,2.04)\end{array}$} & \multirow[t]{2}{*}{0.776} & \multirow{2}{*}{$\begin{array}{l}1.09 \\
(0.59,2.01)\end{array}$} \\
\hline & Group B (GTR\$) & 12.1 & & & & \\
\hline
\end{tabular}

Table 5: Features of the brain metastasis affecting survival in univariate and multivariate analysis (Cox regression model).

$\mathrm{CI}^{*}$ : confidence interval, NA $\dagger$ : not assessed, MCR $\ddagger$ : microscopic complete resection, GTR ${ }^{\S}$ : gross total resection.

A and 23 (53.5\%) in group B ( $\mathrm{p}=0.000)$. In group A, 1-year and 2-year local recurrence was $21.2 \%$ and $25.8 \%$, respectively; and, in group B, $65.2 \%$ for both. In group A, median time-to-progression (TTP) was not reached at median period during follow-up; in group $\mathrm{B}$, median TTP was 8.84 months. PFS curves are in Figure 2.

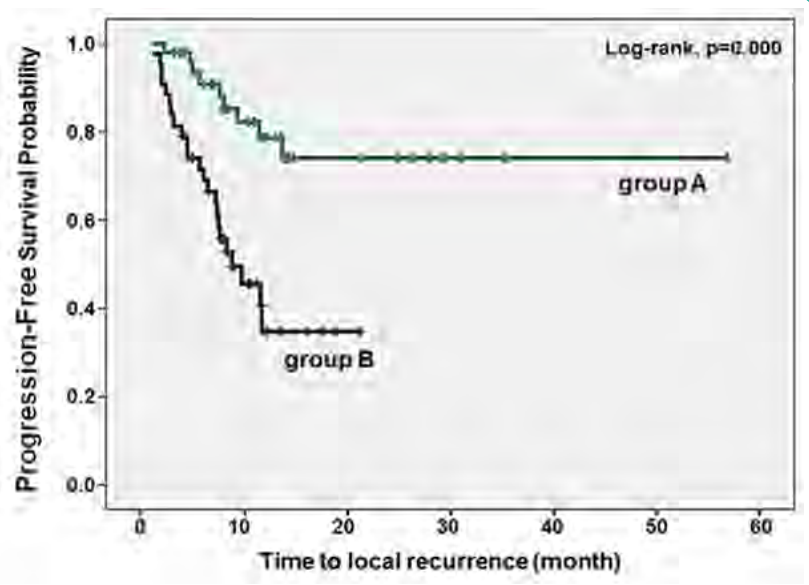

Figure 2: Progression-free survival from local recurrence according to groups $\mathrm{A}$ and $\mathrm{B}$.

By Cox regression analysis, the hazard ratio for local recurrence for group B versus group A was 3.84 (95\% confidence interval, $1.77-8.32 ; \mathrm{p}=0.000)$ in univariate analysis and 3.09 (95\% confidence interval, 1.37-6.96; $\mathrm{p}=0.007)$ in multivariate analysis. In addition to method of surgical resection for BM, we also examined associations between local recurrenceand risk factors of gender, age, pathology, tumor stage, extracranial metastasis, primary tumor status, KPS score, timing of metastasis, tumor size and location, functional grade and postoperative radiotherapy. According to univariate analyses, KPS score was associated with local recurrence but not with local recurrence in multivariate analyses after multifactor adjustment (Tables 7 and 8).

\section{Distant recurrence}

Patterns of distant recurrence by group are in Table 9. A total of 32 patients (35.6\%) had distant recurrences, 21 (44.7\%) of 47 patients in group A and 11 (25.6\%) of 43 patients in group B. Median TTP in group A was 20.3 months.Distant recurrence in group B was not

\begin{tabular}{|l|l|l|l|}
\hline & Total (N = 90) & $\begin{array}{l}\text { group A } \\
(\mathrm{N}=47)\end{array}$ & $\begin{array}{l}\text { group B (N } \\
=43)\end{array}$ \\
\hline Number of patients & $32(35.6 \%)$ & $9(19.1 \%)$ & $23(53.5 \%)$ \\
\hline Mean TTP* (month) & 34.6 & 44.3 & 11.5 \\
\hline Median TTP (month) & NR $\dagger$ & $\mathrm{NR}$ & 8.8 \\
\hline Ranges (month) & $1.2-56.8$ & $2.3-56.8$ & $1.2-21.2$ \\
\hline 1-year recurrence rate & $41.7 \%$ & $21.2 \%$ & $65.2 \%$ \\
\hline 2-year recurrence rate & $44.5 \%$ & $25.8 \%$ & $65.2 \%$ \\
\hline
\end{tabular}

Table 6: Local recurrence analysis and 1-year and 2-year recurrence rates according to group A and $\mathrm{B}$.

TTP*: time-to-progression, $\mathrm{NR}^{\dagger}$ : not reached at median value.

reached at median value during follow-up. PFS curves are in Figure 3. No significant difference in distant recurrence was seen between groups by Kaplan-Meier modeling and Cox regression analysis. The hazard ratio for distant recurrence for patients with well-controlled primary cancer versus those with uncontrolled primary cancer was 2.61 (95\% confidence interval, $1.29-5.26 ; \mathrm{p}=0.005)$ by univariate analysis (Table 10 and 11). Other factors affecting distant recurrence such as primary cancer status and KPS score were associated with distant recurrence in univariate analysis. In multivariate analysis, KPS score was not associated with distant recurrence after multifactor adjustment.

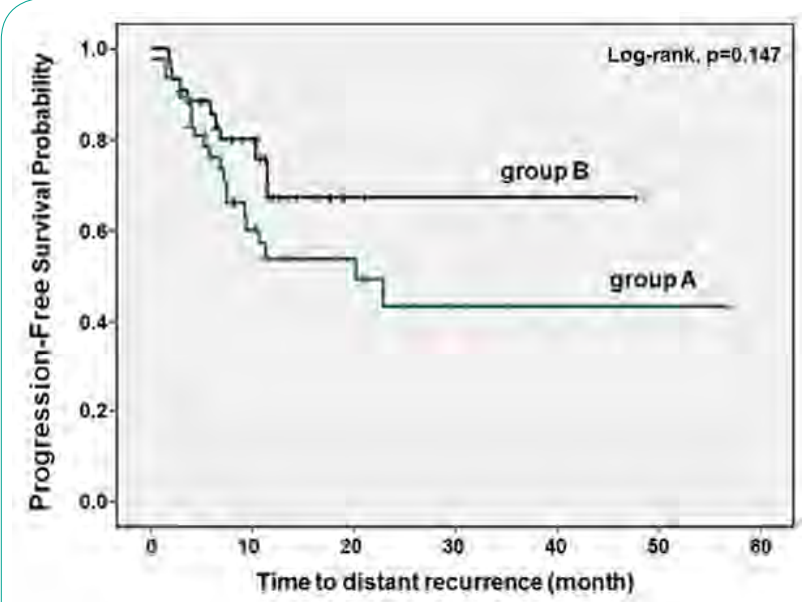

Figure 3: Progression-free survival from distant recurrence according to groups $\mathrm{A}$ and $\mathrm{B}$. 
Citation: Jang JH, Lee YM, Kim YZ (2016) Clinical Benefits of Microscopically Complete Resection in Controlling Local Recurrence of Single Brain Metastasis. Int J Surg Surgical Porced 1: 108. doi: http://dx.doi.org/10.15344/ijssp/2016/108

Page 4 of 9

\begin{tabular}{|c|c|c|c|c|c|c|}
\hline \multicolumn{2}{|l|}{ Factor } & $\begin{array}{l}\text { median } \\
\text { survival } \\
\text { (month) }\end{array}$ & $\begin{array}{l}\text { univariate } \\
\text { analysis } \\
\text { (p value) }\end{array}$ & $\begin{array}{l}\text { Hazard } \\
\text { ratio } \\
\left(95 \% \mathrm{CI}^{*}\right)\end{array}$ & $\begin{array}{l}\text { multivariate } \\
\text { analysis } \\
\text { (p value) }\end{array}$ & $\begin{array}{l}\text { Hazard } \\
\text { ratio } \\
(95 \% \mathrm{CI})\end{array}$ \\
\hline \multirow[t]{2}{*}{ Gender } & male & $\mathrm{NR} \dagger$ & \multirow[t]{2}{*}{0.871} & \multirow{2}{*}{$\begin{array}{l}1.07(0.48, \\
2.38)\end{array}$} & \multirow[t]{2}{*}{$\mathrm{NA}^{*}$} & \\
\hline & female & NR & & & & \\
\hline \multirow[t]{2}{*}{ Age } & $\geq 65$ years & NR & \multirow[t]{2}{*}{0.139} & \multirow{2}{*}{$\begin{array}{l}3.03 \\
(0.78,5.26)\end{array}$} & \multirow[t]{2}{*}{ NA } & \\
\hline & $<65$ years & NR & & & & \\
\hline \multirow[t]{2}{*}{ Pathology } & NSCLC ${ }^{\varsigma}$ & NR & \multirow[t]{2}{*}{0.229} & \multirow{2}{*}{$\begin{array}{l}1.67 \\
(0.72,3.85)\end{array}$} & \multirow[t]{2}{*}{ NA } & \\
\hline & non-NSCLC & NR & & & & \\
\hline \multirow{2}{*}{$\begin{array}{l}\text { Extracranial } \\
\text { metastasis }\end{array}$} & presence & NR & \multirow[t]{2}{*}{0.984} & \multirow{2}{*}{$\begin{array}{l}1.01 \\
(0.48,2.13)\end{array}$} & \multirow[t]{2}{*}{ NA } & \\
\hline & absence & NR & & & & \\
\hline \multirow{2}{*}{$\begin{array}{l}\text { Status of } \\
\text { cancer }\end{array}$} & under-control & NR & \multirow[t]{2}{*}{0.685} & \multirow{2}{*}{$\begin{array}{l}1.16 \\
(0.57,2.34)\end{array}$} & \multirow[t]{2}{*}{ NA } & \\
\hline & out-of-control & NR & & & & \\
\hline \multirow[t]{2}{*}{ KPS $^{\square}$ score } & $\geq 70$ & NR & \multirow[t]{2}{*}{0.040} & \multirow{2}{*}{$\begin{array}{l}2.97 \\
(1.48,5.95)\end{array}$} & \multirow[t]{2}{*}{0.067} & \multirow{2}{*}{$\begin{array}{l}.14 \\
(1.03,4.44)\end{array}$} \\
\hline & $<70$ & 8.3 & & & & \\
\hline \multirow{2}{*}{$\begin{array}{l}\text { Postoperative } \\
\text { radiotherapy }\end{array}$} & performed & NR & \multirow[t]{2}{*}{0.062} & \multirow{2}{*}{$\begin{array}{l}1.60 \\
(0.86,3.06)\end{array}$} & \multirow[t]{2}{*}{0.182} & \multirow{2}{*}{$\begin{array}{l}1.28 \\
(0.63,2.18)\end{array}$} \\
\hline & not performed & 11.6 & & & & \\
\hline
\end{tabular}

Table 7: Clinical characteristics of the patients affecting local recurrence in univariate and multivariate analysis (Cox regression model).

$\mathrm{CI}^{*}$; confidence interval, NR ${ }^{\dagger}$; not reached at median value, NA ${ }^{\ddagger}$; not assessed, NSCLC ${ }^{\varsigma}$; non-small cell lung cancer, KPS ${ }^{\circ}$ Karnofsky Performance Scale.

\begin{tabular}{|c|c|c|c|c|c|c|}
\hline \multicolumn{2}{|l|}{ Factor } & $\begin{array}{l}\text { median } \\
\text { survival } \\
\text { (month) }\end{array}$ & $\begin{array}{l}\text { univariate } \\
\text { analysis } \\
\text { (month) }\end{array}$ & $\begin{array}{l}\text { Hazard } \\
\text { ratio } \\
\left(95 \% \mathrm{CI}^{*}\right)\end{array}$ & $\begin{array}{l}\text { multivariate } \\
\text { analysis } \\
\text { (month) }\end{array}$ & $\begin{array}{l}\text { Hazard } \\
\text { ratio } \\
(95 \% \mathrm{CI})\end{array}$ \\
\hline \multirow{2}{*}{$\begin{array}{l}\text { Timing of } \\
\text { metastasis }\end{array}$} & synchronous & $\mathrm{NR}^{\dagger}$ & \multirow[t]{2}{*}{0.134} & \multirow{2}{*}{$\begin{array}{l}1.79 \\
(0.83,3.87)\end{array}$} & \multirow[t]{2}{*}{$\mathrm{NA}^{\ddagger}$} & \\
\hline & metachronous & NR & & & & \\
\hline \multirow[t]{2}{*}{ Tumor size } & $<3 \mathrm{~cm}$ & $\mathrm{NR}$ & \multirow[t]{2}{*}{0.058} & \multirow{2}{*}{$\begin{array}{l}1.94 \\
(0.97,3.88)\end{array}$} & \multirow[t]{2}{*}{ NA } & \\
\hline & $\geq 3 \mathrm{~cm}$ & NR & & & & \\
\hline \multirow[t]{2}{*}{ Tumor locations } & supratentorial & NR & \multirow[t]{2}{*}{0.055} & \multirow{2}{*}{$\begin{array}{l}3.70 \\
(0.88,15.49)\end{array}$} & \multirow[t]{2}{*}{ NA } & \\
\hline & infratentorial & $\mathrm{NR}$ & & & & \\
\hline \multirow{2}{*}{$\begin{array}{l}\text { Method of } \\
\text { surgery }\end{array}$} & Group A $\left(\mathrm{MCR}^{\S}\right)$ & NR & \multirow[t]{2}{*}{0.000} & \multirow{2}{*}{$\begin{array}{l}3.84 \\
(1.77,8.32)\end{array}$} & \multirow[t]{2}{*}{0.007} & \multirow{2}{*}{$\begin{array}{l}3.09 \\
(1.37,6.96\end{array}$} \\
\hline & Group B (GTR $\left.{ }^{\square}\right)$ & 11.6 & & & & \\
\hline
\end{tabular}

Table 8: Features of the brain metastasis affecting local recurrence in univariate and multivariate analysis (Cox regression model).

$\mathrm{CI}^{*}$; confidence interval, $\mathrm{NR}^{\dagger}$; not reached at median value, $\mathrm{NA}^{*}$; not assessed, $\mathrm{MCR}^{\S}$; microscopic complete resection, $\mathrm{GTR}^{\square}$; gross total resection

\begin{tabular}{|l|l|l|l|}
\hline & $\begin{array}{l}\text { Total } \\
(\mathrm{N}=90)\end{array}$ & $\begin{array}{l}\text { group A } \\
(\mathrm{N}=47)\end{array}$ & $\begin{array}{l}\text { group B } \\
(\mathrm{N}=43)\end{array}$ \\
\hline Number of patients & $32(35.6 \%)$ & $21(44.7 \%)$ & $11(25.6 \%)$ \\
\hline Mean TTP ${ }^{*}$ month) & 32.3 & 29.5 & 34.3 \\
\hline Median TTP (month) & $\mathrm{NR} \dagger$ & 20.3 & $\mathrm{NR}$ \\
\hline Ranges (month) & $0.4-56.8$ & $0.4-56.8$ & $1.9-47.8$ \\
\hline 1-year recurrence rate & $40.2 \%$ & $46.3 \%$ & $33.2 \%$ \\
\hline 2-year recurrence rate & $49.8 \%$ & $57.2 \%$ & $33.2 \%$ \\
\hline
\end{tabular}

Table 9: Distant recurrence analysis and 1-year and 2-year recurrence rates according to group $\mathrm{A}$ and $\mathrm{B}$.

$\mathrm{TTP}^{\star}$; time-to-progression, $\mathrm{NR} \dagger$; not reached at median value.

\section{Discussion}

This study found that local recurrence was reduced by wide resection for BM that included microscopic, infiltrative tumor cells surrounding a tumor with clean surgical margins confirmed by intraoperative margin biopsy. Metastatic brain tumors are commonly circumscribed, although not actually capsulated, and can often be completely separated from neighboring brain tissue. During surgery, after a lesion is located, a cortical incision is made, preferably in the depths of the sulcus overlying the tumor. When the gliotic pseudo capsule is reached, the tumor is usually gradually enucleated from its bed. Although brain metastases appear to be well demarcated from surrounding brain tissue on gross examination, these tumors may have a infiltrative appearance by microscopy $[7,10,11]$. In two autopsy reports, residual tumors were found at the sites of previous surgical excisions in $25 \%$ and $42 \%$ of cases; postoperative radiation was not used in these two series [11,12]. This infiltration is not as extensive as for malignant gliomas. Nevertheless, it may be important in local recurrence after surgical excision. Therefore, we investigated if wide surgical resection for BM including the removal of adjacent tumor cells that infiltrate parenchyma and clean surgical margins confirmed during surgery would establish local cures and diminish local recurrence.

However, we have no data on how much surrounding brain should be suctioned out. In brain metastases, the tumor invasion distance 
Citation: Jang JH, Lee YM, Kim YZ (2016) Clinical Benefits of Microscopically Complete Resection in Controlling Local Recurrence of Single Brain Metastasis. Int J Surg Surgical Porced 1: 108. doi: http://dx.doi.org/10.15344/ijssp/2016/108

Page 4 of 9

\begin{tabular}{|c|c|c|c|c|c|c|}
\hline Factor & median & $\begin{array}{l}\text { median } \\
\text { survival } \\
\text { (month) }\end{array}$ & $\begin{array}{l}\text { univariate } \\
\text { analysis } \\
\text { (p value) }\end{array}$ & $\begin{array}{c}\text { Hazard } \\
\text { ratio } \\
\left(95 \% \mathrm{CI}^{*}\right)\end{array}$ & $\begin{array}{c}\text { multivariate } \\
\text { analysis } \\
\text { (p value) }\end{array}$ & $\begin{array}{l}\text { Hazard } \\
\text { ratio } \\
(95 \% \mathrm{CI})\end{array}$ \\
\hline \multirow[t]{2}{*}{ Gender } & male & $\mathrm{NR}^{\dagger}$ & \multirow[t]{2}{*}{0.419} & \multirow{2}{*}{$\begin{array}{c}1.36 \\
(0.64,2.87)\end{array}$} & \multirow[t]{2}{*}{$\mathrm{NA}^{\ddagger}$} & \\
\hline & female & NR & & & & \\
\hline \multirow[t]{2}{*}{ Age } & $\geq 65$ years & NR & \multirow[t]{2}{*}{0.397} & \multirow{2}{*}{$\begin{array}{c}1.46 \\
(0.60,3.56)\end{array}$} & \multirow[t]{2}{*}{ NA } & \\
\hline & $<65$ years & NR & & & & \\
\hline \multirow[t]{2}{*}{ Pathology } & NSCLC & NR & \multirow[t]{2}{*}{0.734} & \multirow{2}{*}{$\begin{array}{c}1.14 \\
(0.54,2.40)\end{array}$} & \multirow[t]{2}{*}{ NA } & \\
\hline & non-NSCLC & NR & & & & \\
\hline \multirow{2}{*}{$\begin{array}{l}\text { Extracranial } \\
\text { metastasis }\end{array}$} & presence & NR & \multirow[t]{2}{*}{0.083} & \multirow{2}{*}{$\begin{array}{c}1.89 \\
(0.91,3.62)\end{array}$} & \multirow[t]{2}{*}{ NA } & \\
\hline & absence & NR & & & & \\
\hline \multirow{2}{*}{$\begin{array}{l}\text { Status of } \\
\text { cancer }\end{array}$} & under-control & NR & \multirow[t]{2}{*}{0.005} & \multirow{2}{*}{$\begin{array}{c}2.61 \\
(1.29,5.26)\end{array}$} & \multirow[t]{2}{*}{0.023} & \multirow{2}{*}{$\begin{array}{c}2.89 \\
(1.16,7.23)\end{array}$} \\
\hline & out-of-control & 11.4 & & & & \\
\hline \multirow[t]{2}{*}{$\mathrm{KPS}^{\square}$ score } & $\geq 70$ & NR & \multirow[t]{2}{*}{0.034} & \multirow{2}{*}{$\begin{array}{c}2.70 \\
(1.04,7.03)\end{array}$} & \multirow[t]{2}{*}{0.863} & \multirow{2}{*}{$\begin{array}{c}1.20 \\
(0.15,9.94)\end{array}$} \\
\hline & $<70$ & NR & & & & \\
\hline \multirow[t]{2}{*}{ Postoperative radiotherapy } & performed & NR & \multirow[t]{2}{*}{0.278} & \multirow{2}{*}{$\begin{array}{c}1.55 \\
(0.70,3.46)\end{array}$} & \multirow[t]{2}{*}{0.383} & \multirow{2}{*}{$\begin{array}{c}1.58 \\
(0.57,4.38)\end{array}$} \\
\hline & not performed & 11.6 & & & & \\
\hline
\end{tabular}

Table 10: Clinical characteristics of the patients affecting distant recurrence in univariate and multivariate analysis (Cox regression model).

$\mathrm{CI}^{*}$; confidence interval, NR†; not reached at median value, NA ; not assessed, NSCLC\$; non-small cell lung cancer, KPS ; Karnofsky Performance Scale.

\begin{tabular}{|c|c|c|c|c|c|c|}
\hline Factor & median & $\begin{array}{l}\text { median } \\
\text { survival } \\
\text { (month) }\end{array}$ & $\begin{array}{l}\text { univariate } \\
\text { analysis } \\
\text { (month) }\end{array}$ & $\begin{array}{c}\text { Hazard } \\
\text { ratio } \\
\left(95 \% \mathrm{CI}^{*}\right)\end{array}$ & $\begin{array}{c}\text { multivariate } \\
\text { analysis } \\
\text { (month) }\end{array}$ & $\begin{array}{l}\text { Hazard } \\
\text { ratio } \\
(95 \% \mathrm{CI})\end{array}$ \\
\hline \multirow[t]{2}{*}{ Timing of metastasis } & synchronous & $\mathrm{NR}^{\dagger}$ & \multirow[t]{2}{*}{0.458} & \multirow{2}{*}{$\begin{array}{c}1.30 \\
(0.65,2.62)\end{array}$} & \multirow[t]{2}{*}{$\mathrm{NA}^{*}$} & \\
\hline & metachronous & NR & & & & \\
\hline \multirow[t]{2}{*}{ Tumor size } & $<3 \mathrm{~cm}$ & NR & \multirow[t]{2}{*}{0.570} & \multirow{2}{*}{$\begin{array}{c}1.24 \\
(0.59,2.62)\end{array}$} & \multirow[t]{2}{*}{ NA } & \\
\hline & $\geq 3 \mathrm{~cm}$ & NR & & & & \\
\hline \multirow[t]{2}{*}{ Tumor locations } & supratentorial & NR & \multirow[t]{2}{*}{0.230} & \multirow{2}{*}{$\begin{array}{c}2.04 \\
(0.62,6.70)\end{array}$} & \multirow[t]{2}{*}{ NA } & \\
\hline & infratentorial & NR & & & & \\
\hline \multirow{2}{*}{$\begin{array}{l}\text { Method of } \\
\text { surgery }\end{array}$} & Group A $\left(\mathrm{MCR}^{\S}\right)$ & NR & \multirow[t]{2}{*}{0.071} & \multirow{2}{*}{$\begin{array}{c}1.94 \\
(0.93,4.02)\end{array}$} & \multirow[t]{2}{*}{0.173} & \multirow{2}{*}{$\begin{array}{c}1.87 \\
(0.76,4.59)\end{array}$} \\
\hline & Group B (GTR $\left.{ }^{\square}\right)$ & NR & & & & \\
\hline
\end{tabular}

Table 11: Features of the brain metastasis affecting distant recurrence in univariate and multivariate analysis (Cox regression model).

$\mathrm{CI}^{*}$; confidence interval, NR $\dagger$; not reached at median value, NA ; not assessed, MCR\$; microscopic complete resection, GTR ${ }^{\square}$; gross total resection.

usually is reported as not $>5 \mathrm{~mm}$, as described in the literature. Thus, we tried to reset surrounding brain tissue to at least $5 \mathrm{~mm}$ in depth. Actually, we do not have any statistically accurate data on how many frozen sections of the tumor margin were positive for cancer, although up to $\sim 10-15 \%$ of the biopsy samples were positive for tumor cells. In each case, our pathologists studied 6-10 margin biopsy samples.

Although many studies have addressed factors that influence therapeutic results when $\mathrm{BM}$ from lung cancer is treated using different therapeutic modalities, no comprehensive study has assessed the influence of surgical extent on recurrence and survival in patients with BM.The incidence of local recurrence after BM excision is around $40 \%$ in many series $[6,13]$. However, our study observed only 9 recurrences $(19.1 \%)$ at a site treated by tumor resection with tumor cells infiltrating adjacent brain parenchyma, even without postoperative brain irradiation. The reason for this successful local control was attributed to the absence of remnant tumor burden due to microscopically complete resection, including microscopic, infiltrative tumor cells adjacent to brain parenchyma.
Despite the local control rate achieved in this study, distant CNS relapse rate was as high as $44.7 \%$ in patients who underwent microscopic complete resection, similar to other studies $[2,14]$. Median survival was 13.1 months. Reported survival after surgery for $\mathrm{BM}$ ranges from 8 to 20 months [15-20]. We observed little difference in survival between the two groups (median survival time; 13.1 months in group A vs. 12.1 months in group $B, p=0.309$ ). Patients with higher performance status at presentation had longer survival. This study showed that excellent local control of BM, although necessary for prolonged survival, is not sufficient to ensure long-term survival.

Long-term survival will be achieved only when concurrent advances are made in treatment of local and distant CNS disease and systemic cancer. Relevant to this point, Macchiarini et al. showed that systemic chemotherapy is the most significant independent predictor of disease-free long-term survival after neurosurgical resection of BM [21]. Many studies found systemic chemotherapy to be an important therapeutic modality that offers therapeutic benefits to patients with BM [22-25]. In our study, a few CNS-related deaths occurred, most 
Citation: Jang JH, Lee YM, Kim YZ (2016) Clinical Benefits of Microscopically Complete Resection in Controlling Local Recurrence of Single Brain Metastasis. Int J Surg Surgical Porced 1: 108. doi: http://dx.doi.org/10.15344/ijssp/2016/108

Page 4 of 9

attributable to primary cancer progression. Thus,for patients with $\mathrm{BM}$, our findings indicated that early and optimal therapy for primary cancer is critically needed to prevent death.

The goal of treatment for $\mathrm{BM}$ is to eliminate the metastasis and prevent tumor recurrence in the brain. For patients with BM, despite substantial advances in local control, treatment of primary cancer is most important for prolonging overall survival [26]. In our study, factors influencing survival were not features of brain metastasis, but related to patients' clinical condition. Our results also showed that poorly controlled primary cancer had higher distant recurrence rates than well-controlled primary tumors; the only factor affecting distant recurrence was primary tumor status. Therefore, to prolong survival and reduce distant recurrence, controlling systemic disease is the most important. Thus, one of the most important advantages of microscopically complete resection is that it allows systemic chemotherapy to be started sooner. This could allow control of both the primary cancer and BM and may establish a local cure or complete remission of brain disease.

In terms of radiotherapy for brain metastasis, class I evidence indicates that radiation therapy is the optimal choice for patients with metastatic brain tumors. We also used radiotherapy for the patients with brain metastases, especially located in the eloquent and critical area but not in routine practice. In some cases of NSCLC, however, we initially performed systemic chemotherapy postoperatively for the early control of the primary disease. Brain irradiation was delayed until a local cerebral recurrence developed. In cases of small, deepseated, surgically inaccessible tumors, uncontrolled primary disease, or a KPS score too poor for general anesthesia, we also performed radiosurgery.

\section{Conclusion}

The present study provides the first published description of surgical outcomes for wide surgical resection of BM including microscopically infiltrative cancer cells adjacent to brain parenchyma with clean surgical margins confirmed by intraoperative margin biopsy. This study showed that wide surgical resection of BM with clean surgical margins confirmed during surgery could reduce local recurrence.

\section{Acknowledgments}

The authors thank Young Min Kim, M.D. and Mi-Ok Sunwoo, M.D. (Department of Radiology, Samsung Changwon Hospital) for their review of the neuroradiological images; Eun Hee Lee, M.D., and Mee-Seon Kim, M.D. (Department of Pathology, Samsung Changwon Hospital) for their pathological diagnosis.

\section{Competing Interests}

The authors declare that they have no competing interests.

\section{Funding}

This study was financially supported by a Samsung Biomedical Research Institute grant (SMR-112061). The authors report no conflict of interest concerning the materials or methods used in this study or the findings specified in this paper.

\section{References}

1. Larson D, Rubenstein J, McDermott M (2004) Treatment of metastatic cancer, in DeVitaVT Jr, Hellman S, Rosenberg SA (eds). Cancer Principles and Practice of Oncology. Philadelphia: Lippincott Williams \& Wilkins 23232398.

2. Patchell RA, Tibbs PA, Walsh JW, Dempsey RJ, Maruyama Y, et al. (1990) A randomized trial of surgery in the treatment of single metastases to the brain. N Engl J Med 322: 494-500.

3. Lang Frederick F, Chang Eric L, Abis-Said Dima, Wildrick David M, Sawaya Raymond (2003) Metastatic brain tumors in Youmans JR (ed.): Neurological Surgery, ed 5. Philadelphia: WB Saunders 1: 1077-1097.

4. Stark AM, Tscheslog H, Buhl R, Held-Feindt J, Mehdorn HM (2005) Surgical treatment for brain metastases: prognostic factors and survival in 177 patients. Neurosurg Rev 28: 115-119.

5. Paek SH, Audu PB, Sperling MR, Cho J, Andrews DW (2005) Reevaluation of surgery for the treatment of brain metastases: review of 208 patients with single or multiple brain metastases treated at one institution with modern neurosurgical techniques. Neurosurgery 56: 1021-1034.

6. Sundaresan N, Sachdev VP, DiGiacinto GV, Hughes JE (1988) Reoperation for brain metastases. J Clin Oncol 6: 1625-1629.

7. Sundaresan N, Galicich JH (1985) Surgical treatment of brain metastases Clinical and computerized tomography evaluation of the results of treatment. Cancer 55: 1382-1388.

8. Gaspar L, Scott C, Rotman M, Asbell S, Phillips T, et al. (1997) Recursive partitioning analysis (RPA) of prognostic factors in three Radiation Therapy Oncology Group (RTOG) brain metastases trials. Int J Radiat Oncol Biol Phys 37: 745-751.

9. Sawaya R, Hammoud M, Schoppa D, Hess KR, Wu SZ, et al. (1998) Neurosurgical outcomes in a modern series of 400 craniotomies for treatment of parenchymal tumors. Neurosurgery 42: 1044-1055.

10. Henson R, Urich H (1982) (Eds.) Cancer and Nervous System, London: Blackwell.

11. STORTEBECKER TP (1954) Metastatic tumors of the brain from a neurosurgical point of view; a follow-up study of 158 cases. J Neurosurg 11: 84-111.

12. Lang EF, Slater J (1964) METASTATIC BRAIN TUMORS. RESULTS OF SURGICAL AND NONSURGICAL TREATMENT. Surg Clin North Am 44: 865-872.

13. Sundaresan N, Galicich JH (1985) Surgical treatment of single brain metastases from non-small-cell lung cancer. Cancer Invest 3: 107-113.

14. Patchell RA, Tibbs PA, Regine WF, Dempsey RJ, Mohiuddin M, et al (1998) Postoperative radiotherapy in the treatment of single metastases to the brain: a randomized trial. JAMA 280: 1485-1489.

15. Hankins JR, Miller JE, Salcman M, Ferraro F, Green DC, et al. (1988) Surgical management of lung cancer with solitary cerebral metastasis. Ann Thorac Surg 46: 24-28.

16. Magilligan DJ Jr, Duvernoy C, Malik G, Lewis JW Jr, Knighton R, et al. (1986) Surgical approach to lung cancer with solitary cerebral metastasis: twenty-five years' experience. Ann Thorac Surg 42: 360-364.

17. Ewend MG, Brem S, Gilbert M, Goodkin R, Penar PL, et al. (2007) Treatment of single brain metastasis with resection, intracavity carmustine polymer wafers, and radiation therapy is safe and provides excellent local control. Clin Cancer Res 13: 3637-3641.

18. Mussi A, Pistolesi M, Lucchi M, Janni A, Chella A, et al. (1996) Resection of single brain metastasis in non-small-cell lung cancer: prognostic factors. $J$ Thorac Cardiovasc Surg 112: 146-153

19. Salvati M Cervoni L, Delfini R (1996) Solitary brain metastases from nonoat cell lung cancer: clinical and prognostic features. Neurosurg Rev 19: 221-225

20. Wroß,,ski M, Arbit E, Burt M, Galicich JH (1995) Survival after surgical treatment of brain metastases from lung cancer: a follow-up study of 231 patients treated between 1976 and 1991. J Neurosurg 83: 605-616. 
Citation: Jang JH, Lee YM, Kim YZ (2016) Clinical Benefits of Microscopically Complete Resection in Controlling Local Recurrence of Single Brain Metastasis. Int J Surg Surgical Porced 1: 108. doi: http://dx.doi.org/10.15344/ijssp/2016/108

21. Macchiarini P, Buonaguidi R, Hardin M, Mussi A, Angeletti CA (1991) Results and prognostic factors of surgery in the management of non-small cell lung cancer with solitary brain metastasis. Cancer 68: 300-304.

22. Postmus PE, Smit EF (1999) Chemotherapy for brain metastases of lung cancer: a review. Ann Oncol 10: 753-759.

23. Soffietti R, Costanza A, Laguzzi E, Nobile M, Rudà R (2005) Radiotherapy and chemotherapy of brain metastases. J Neurooncol 75: 31-42.

24. van den Bent MJ (2003) The role of chemotherapy in brain metastases. Eur J Cancer 39: 2114-2120.

25. Schuette W (2004) Treatment of brain metastases from lung cancer: chemotherapy. Lung Cancer 45 Suppl 2: S253-257.

26. Andrews DW, Scott CB, Sperduto PW, Flanders AE, Gaspar LE, et al. (2004) Whole brain radiation therapy with or without stereotactic radiosurgery boost for patients with one to three brain metastases: phase III results of the RTOG 9508 randomised trial. Lancet 363: 1665-1672. 